\title{
Study of Critical Sets in Latin Squares by using the Autotopism Group
}

\author{
R. M. Falcón ${ }^{1}$ \\ Departamento de Matemática Aplicada I. \\ E. T. S. de Arquitectura. Universidad de Sevilla. \\ Avda. Reina Mercedes 2. 41012-Sevilla (Spain).
}

\begin{abstract}
Given a Latin square $L$ and a subset $\mathfrak{F}$ of its autotopism group $\mathcal{U}(L)$, we study in this paper some properties and results which partial Latin squares contained in $L$ inherit from $\mathcal{U}(L)$, by using $\mathfrak{F}$. In particular, we define the concept of $\mathfrak{F}$-critical set of $L$ and we ask ourselves about the smallest one contained in $L$.
\end{abstract}

Keywords: Latin Square, Autotopism Group.

\section{Introduction}

A Latin square $L$ of order $n$ is a $n \times n$ array with elements chosen from the set $N=\{0,1, \ldots, n-1\}$, such that each symbol occurs precisely once in each row and each column. The set of Latin squares of order $n$ is denoted by $L S(n)$. If $L=\left(l_{i, j}\right) \in L S(n)$, the orthogonal array representation of $L$ is the set of $n^{2}$ triples $\left\{\left(i, j, l_{i, j}\right): i, j \in N\right\}$. The previous set is identified with $L$ and so, it is written $\left(i, j, l_{i, j}\right) \in L$, for all $i, j \in N$. It is said that $L$ is an entropic Latin square if $l_{l_{i j} l_{s t}}=l_{l_{i s} l_{j t}}$, for all $i, j, s, t \in N$.

$\overline{1}$ Email: rafalgan@us.es 
Let $S_{n}$ be the symmetric group on $N$. An isotopism of Latin squares of order $n$ is then a triple $\Theta=(\alpha, \beta, \gamma) \in \mathcal{I}_{n}=S_{n} \times S_{n} \times S_{n}$. If we apply $\Theta$ to a Latin square $L \in L S(n)$, it is verified that $\alpha, \beta$ and $\gamma$ are respectively, permutations of rows, columns and symbols of $L$. The resulting square $L^{\Theta}$ is also a Latin square and it is said to be isotopic to $L$. In particular, if $L=\left(l_{i, j}\right)$, then $L^{\Theta}=\left\{\left(i, j, \gamma\left(l_{\alpha^{-1}(i), \beta^{-1}(j)}\right): 0 \leq i, j \leq n-1\right\}\right.$. An isotopism which maps $L$ to itself is an autotopism. The stabilizer subgroup of $L$ in $S_{n}^{3}$ is its autotopism group, $\mathcal{U}(L)=\left\{\Theta \in \mathcal{I}_{n}: L^{\Theta}=L\right\}$.

A partial Latin square, $P$, of order $n$, is a $n \times n$ array with elements chosen from a set of $n$ symbols, such that each symbol occurs at most once in each row and in each column. The set of partial Latin squares of order $n$ is denoted by $P L S(n)$. The size of $P,|P|$, is the number of non-blank cells. If $L \in L S(n)$ we will denote by $L_{i, j}$ the partial Latin square contained in $L$ such that the unique filled cell of $L_{i, j}$ is $\left(i, j, l_{i, j}\right)$. Thus, given $P \in P L S(n)$ we can ever find a subset $I_{P} \subseteq N \times N$ such that $P=\bigcup_{(i, j) \in I_{P}} L_{i, j}$.

It is said that $P$ can be completed to a Latin square $L \in L S(n)$ if $P \subseteq L$. If $L$ is the unique one in such conditions, it is said that $P$ is uniquely completable to $L$ and it is denoted $P \in U C(L)$. If besides any proper subset of $P$ can be completed at least to two distinct Latin squares it is said that $P$ is a critical set of $L$ and it is denoted $P \in C S(L)$. Given $L \in L S(n), \operatorname{scs}(L)$ denotes the size of the smallest critical set of $L$ and $\operatorname{scs}(n)$ denotes the minimum of $\operatorname{scs}(L)$ for all $L \in L S(n)$. Analogously, $l \operatorname{cs}(L)$ denotes the size of the largest critical set of $L$ and $l \operatorname{cs}(n)$ denotes the maximum of $l c s(L)$ for all $L \in L S(n)$.

\section{Extended autotopisms of partial Latin squares}

An isotopism of partial Latin squares of order $n$ will be a triple $\Theta=(\alpha, \beta, \gamma) \in$ $\mathcal{I}_{n}$, where $\gamma(\emptyset)=\emptyset$.

Lemma 2.1 Let $P \in P L S(n)$ be contained in a Latin square $L \in L S(n)$ and let $\Theta \in \mathcal{I}_{n}$. The following asserts are verified:

a) $P^{\Theta}$ is also in $P L S(n)$ and $\left|P^{\Theta}\right|=|P|$,

b) If $Q \in P L S(n)$ verifies that $P \subseteq Q$, then $P^{\Theta} \subseteq Q^{\Theta}$.

c) If $P$ can be completed to $L$, then $P^{\Theta}$ can be completed to $L^{\Theta}$.

Lemma 2.2 Given $L \in L S(n)$, let $\Theta_{1}, \Theta_{2} \in \mathcal{U}(L)$ be two distinct autotopisms of $L$. Let us consider $L_{i_{1}, j_{1}}, L_{i_{2}, j_{2}} \in P L S(n)$ with $\left(i_{1}, j_{1}\right) \neq\left(i_{2}, j_{2}\right)$. Then $L_{i_{1}, j_{1}}^{\Theta_{1}} \neq L_{i_{2}, j_{2}}^{\Theta_{1}}$ and $L_{i_{1}, j_{1}}^{\Theta_{1}} \neq L_{i_{1}, j_{1}}^{\Theta_{2}}$. 
Now, let us consider $L \in L S(n)$ and let $\mathfrak{F} \subseteq \mathcal{U}(L)$. If $P \in P L S(n)$ can be completed to $L$, we will define $P^{\mathfrak{F}} \in P L S(n)$ as $P^{\mathfrak{F}}=\bigcup_{\Theta \in \mathfrak{F}} P^{\Theta}$. Then, we will say that $P^{\mathfrak{F}}$ is an extended autotopy of $P$.

Lemma 2.3 Let us suppose $L \in L S(n), P \in P L S(n)$ contained in $L$ and $\mathfrak{F} \subseteq \mathcal{U}(L)$. Then, $\left|P^{\mathfrak{F}}\right| \leq|P| \cdot|\mathfrak{F}|$.

Example 2.4 Let $L=\left(\begin{array}{ll}0 & 1 \\ 1 & 0\end{array}\right) \in L S(2)$. So, $\mathcal{U}(L)=\{(I d, I d, I d),((I d,(01)$, $(01)),((01), I d,(01)),((01),(01), I d)\}$. Let us take now by example $\mathfrak{F}=$ $\{(I d, I d, I d),(I d,(01),(01))\}, P=\left(\begin{array}{c}0 \\ * *\end{array}\right) \in P L S(2)$ and $Q=\left(\begin{array}{ll}0 & * \\ * & 1\end{array}\right) \in P L S(2)$. Then, we can prove that $P^{\mathfrak{F}}=\left(\begin{array}{cc}0 & 1 \\ * *\end{array}\right)$ and $P^{\mathcal{U}(L)}=L=Q^{\mathfrak{F}}=Q^{\mathcal{U}(L)}$.

In general, given $L \in L S(n)$, there does not exist a subset $\mathfrak{F}$ of $\mathcal{U}(L)$ and $P \in P L S(n)$ such that $P \subset L$ and $P^{\mathfrak{F}}=L$. This is due to that the most of Latin squares has only the trivial autotopism group [1], $\mathcal{U}(L)=\{(I d, I d, I d)\}$. We can therefore ask ourselves about conditions under which we can obtain a similar result to Example 2.4:

Theorem 2.5 Every entropic Latin square is an extended autotopy of each of its proper partial Latin squares.

Example 2.6 Let $L=\left(\begin{array}{lll}0 & 1 & 2 \\ 1 & 2 & 0 \\ 2 & 0 & 1\end{array}\right) \in L S(3)$. It is entropic and verifies that $|\mathcal{U}(L)|=18$ [1]. Let $\mathfrak{F}=\left\{\left(\alpha_{s}, \alpha_{t}, \alpha_{l_{s t}}\right)\right\}_{s, t \in N}$, where $\alpha_{0}=I d, \alpha_{1}=(012), \alpha_{2}=$ (021). So, $|\mathfrak{F}|=9$ and $P^{\Theta}=L$ for all $P \in P L S(3)$ contained in $L$. $\quad \triangleleft$

\section{Critical sets by considering $\mathcal{U}(L)$}

Given $L \in L S(n)$ and $\mathfrak{F} \subseteq \mathcal{U}(L)$, let $<\mathfrak{F}>$ be the subgroup of $\mathcal{U}(L)$ generated by composing the elements of $\mathfrak{F} \cup \mathfrak{F}^{-1}$, where $\mathfrak{F}^{-1}=\left\{\Theta^{-1}=\left(\alpha^{-1}, \beta^{-1}, \gamma^{-1}\right)\right.$ : $\Theta=(\alpha, \beta, \gamma) \in \mathfrak{F}\} \subseteq \mathcal{U}(L)$. Now, given $P \in P L S(n)$ contained in $L$, let us denote by $\mathfrak{F}(P)$ the partial Latin square $P^{<\mathfrak{F}>}$. We will then say that $P$ is uniquely $\mathfrak{F}$-completable to $L$ and it will be denoted by $P \in U C_{\mathfrak{F}}(L)$ if $\mathfrak{F}(P) \in U C(L)$. We will say that $C$ is a $\mathfrak{F}$-critical set of $L$ if $C \in U C_{\mathfrak{F}}(L)$ and $P \notin U C_{\mathfrak{F}}(L)$ for all $P \subset C$. So, we are interested in the smallest size $\operatorname{scs}_{\mathfrak{F}}(L)$ of a $\mathfrak{F}$-critical set of $L$.

Lemma 3.1 Let $L \in L S(n)$. The next assertions are verified:

a) Given $\mathfrak{F}_{1}, \mathfrak{F}_{2} \subseteq \mathcal{U}(L)$ such that $\mathfrak{F}_{1} \subseteq \mathfrak{F}_{2}$, then $\operatorname{scs}_{\mathfrak{F}_{2}}(L) \leq \operatorname{scs}_{\mathfrak{F}_{1}}(L)$.

b) If $\mathfrak{F} \subseteq \mathcal{U}(L)$ is such that $|\langle\mathfrak{F}\rangle| \geq \operatorname{lcs}(L)$, then $\operatorname{scs}_{\mathfrak{F}}(L)=1$. 
Lemma 3.2 Let $L \in L S(n), P \in P L S(n)$ contained in $L$ and $\mathfrak{F} \subseteq \mathcal{U}(L)$. Let $C \in C S(L)$ be such that $|C|=\operatorname{scs}(L)$. If $C \subseteq \mathfrak{F}(P)$ then, scs $\mathfrak{F}(L) \leq|P|$. So, $\operatorname{scs}_{\mathfrak{F}}(L) \leq \operatorname{scs}(L)$, for all $\mathfrak{F} \subseteq \mathcal{U}(L)$, such that $\mathfrak{F} \neq \emptyset$.

Proposition 3.3 Let $L \in L S(n), P \in P L S(n)$ contained in $L$ and $\mathfrak{F} \subseteq \mathcal{U}(L)$. Then, $\operatorname{scs}_{\mathfrak{F}}(L)=\min _{P \in P L S(n)}\{|P|: \exists C \in C S(L)$ such that $C \subseteq \mathfrak{F}(P)\}$.

Example 3.4 Let $L=\left(\begin{array}{ccccc}0 & 1 & 2 & 3 & 4 \\ 1 & 2 & 3 & 4 & 0 \\ 2 & 3 & 4 & 0 & 1 \\ 3 & 4 & 0 & 1 & 2 \\ 4 & 0 & 1 & 2 & 3\end{array}\right) \in L S(5), C=\left(\begin{array}{ccccc}0 & 1 & * & * & * \\ 1 & * & * & * & * \\ * & * & * & * & * \\ * & * & * & * & * \\ * & * & * & 2 & 2\end{array}\right) \in C S(L)$ and $P=$

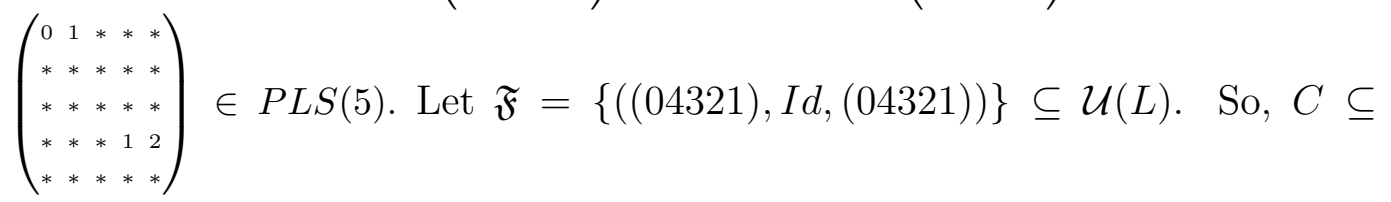

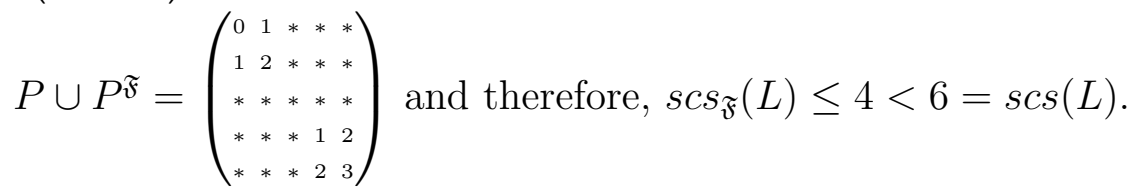

3.1 An algorithm to obtain an upper bound of $\operatorname{scs}_{\mathfrak{F}}(L)$

Lemma 3.5 Let $L=\left(l_{i j}\right) \in L S(n), \mathfrak{F} \subseteq \mathcal{U}(L), P \in P L S(n)$ be contained in $L$ such that $|P|=\operatorname{scs}_{\mathfrak{F}}(L)$ and $C \in C S(L)$ be contained in $\mathfrak{F}(P)$. For all $i, j \in N$, there exist $\left(s, t, l_{s t}\right) \in C$ and $\Theta \in<\mathfrak{F}>$ such that $L_{i, j}^{\Theta}=L_{s, t}$.

Lemma 3.6 Let $L \in L S(n), \mathfrak{F} \subseteq \mathcal{U}(L), P \in P L S(n)$ be contained in $L$ such that $|P|=\operatorname{scs}_{\mathfrak{F}}(L)$ and $C \in C S(L)$ be contained in $\mathfrak{F}(P)$. Then $P \subseteq \mathfrak{F}(C)$.

In general, given $L=\left(l_{i j}\right) \in L S(n), P=\bigcup_{(i, j) \in I_{P}} L_{i, j} \in P L S(n)$ contained in $L$ and $\mathfrak{F} \subseteq \mathcal{U}(L)$, we must be interested in an algorithm which allows us to obtain the number $\operatorname{scs}_{\mathfrak{F}}(L)$. To do it, let $\mathfrak{F}(P)=\bigcup_{(i, j) \in I_{\mathfrak{F}(P)}} L_{i, j} \in P L S(n)$, which is contained in $L$. Given $\left(i, j, l_{i j}\right) \in \mathfrak{F}(P)$, let us consider:

$$
S_{i, j}^{P}=\left\{\left(s, t, l_{s t}\right) \in P \text { such that } L_{i, j} \subseteq \mathfrak{F}\left(L_{s, t}\right)\right\} \subseteq P .
$$

Lemma 3.7 Let $L \in L S(n), P, Q \in P L S(n)$ be both contained in $L$ and $\mathfrak{F} \subseteq \mathcal{U}(L)$. If $Q \subseteq \mathfrak{F}(P)$ and $P \subseteq \mathfrak{F}(Q)$, then $P=\bigcup_{(i, j) \in I_{Q}} S_{i, j}^{P}$ and $Q=$ $\bigcup_{(i, j) \in I_{P}} S_{i, j}^{Q}$.

Theorem 3.8 Let $L \in L S(n)$ and $\mathfrak{F} \subseteq \mathcal{U}(L)$. Then $\operatorname{scs}_{\mathfrak{F}}(L)$ is equal to:

$$
\min _{C \in C S(L)}\left\{\min \left\{|P|: \exists I_{P} \subseteq I_{\mathfrak{F}(C)} \text { being } P=\bigcup_{(i, j) \in I_{P}} L_{i, j}, C=\bigcup_{(i, j) \in I_{P}} S_{i, j}^{C}\right\}\right\} .
$$


Proof. Let $C \in C S(L)$ and $I_{P} \subseteq I_{\mathfrak{F}(C)}$ be such that $C=\bigcup_{(i, j) \in I_{P}} S_{i, j}^{C}$, being $P=\bigcup_{(i, j) \in I_{P}} L_{i, j} \subseteq \mathfrak{F}(C)$. So, given $\left(s, t, l_{s t}\right) \in C$, there exists $\Theta \in\langle\mathfrak{F}>$ and $(i, j) \in I_{P}$ such that $L_{s, t}^{\Theta}=L_{i, j}$. Then, $L_{i, j}^{\Theta^{-1}}=L_{s, t}$ and so, $\left(s, t, l_{s t}\right) \in$ $\mathfrak{F}^{-1}(P)=\mathfrak{F}(P)$. Therefore, we have that $C \subseteq \mathfrak{F}(P)$. So, from Proposition $3.3, \operatorname{scs}_{\mathfrak{F}}(L)$ is smaller than the signaled minimum. Now, let $P \in P L S(n)$ contained in $L$ be such that $|P|=\operatorname{scs}_{\mathfrak{F}}(L)$. From Proposition 3.3, there exist $C \in C S(L)$ contained in $\mathfrak{F}(P)$. Besides, from Lemma 3.6, $P \subseteq \mathfrak{F}(C)$. So, Lemma 3.7 involves $C=\bigcup_{(i, j) \in I_{P}} S_{i, j}^{C}$, being $I_{P} \subseteq I_{\mathfrak{F}(C)}$, and therefore, by using Proposition 3.3 again, $\operatorname{scs}_{\mathfrak{F}}(L)$ is bigger than the signaled minimum.

The computation of the minimum of the previous theorem allows us to obtain $\operatorname{scs}_{\mathfrak{F}}(L)$ but it can be an arduous process. In a concrete case, a first upper bound of $\operatorname{scs}_{\mathfrak{F}}(L)$ can be given by the following way: let $C \in C S(L)$ be such that $|C|=\operatorname{scs}(L)$. Let us obtain $\mathfrak{F}(C)$ and next all the sets $S_{i, j}^{C}$. If the cardinality of all these sets is one, then we cannot improve the upper bound of $\operatorname{scs}(L)$ by using this critical set $C$. In the other case, let $n_{1}>1$ be the maximum of the mentioned cardinalities and let us take $S_{i_{1}, j_{1}}^{C}$ a set with cardinality $n_{1}$. Let us now fixe $\left(s_{1}, t_{1}, l_{s_{1} t_{1}}\right) \in S_{i_{1}, j_{1}}^{C}$ and let $\Theta_{1} \in \mathfrak{F}$ be such that $L_{s_{1}, t_{1}}^{\Theta_{1}}=L_{i_{1}, j_{1}}$. Let us then consider $C_{1}=C \backslash\left(S_{i_{1}, j_{1}}^{C} \backslash\left\{\left(s_{1}, t_{1}, l_{s_{1} t_{1}}\right)\right\}\right)$ and $P_{1}=C_{1}^{\Theta_{1}} \subseteq C^{\Theta_{1}}$. So, $\left|P_{1}\right|=\left|C_{1}\right|<|C|$ and it can be seen that $C \subseteq \mathfrak{F}\left(P_{1}\right)$ and therefore, $\operatorname{scs}_{\mathfrak{F}}(L) \leq\left|C_{1}\right|<\operatorname{scs}(L)$. Now we can take the same procedure with $C_{1}$ instead of $C$. If the cardinality of all the corresponding $S_{i, j}^{C_{1}}$ is one, then we cannot improve the upper bound of $\operatorname{scs}_{\mathfrak{F}}(L)$ by using this method with $C$. In the other case, we take $S_{i_{2}, j_{2}}^{C_{1}}$ a set with cardinality $n_{2}>1$, the maximum of the mentioned cardinalities, and we fixe $\left(s_{2}, t_{2}, l_{s_{2} t_{2}}\right) \in S_{i_{2}, j_{2}}^{C_{1}}$. Let us observe that it is necessary to take $\left(s_{2}, t_{2}, l_{s_{2} t_{2}}\right)=\left(s_{1}, t_{1}, l_{s_{1} t_{1}}\right)$ whenever it is possible. So, we consider $C_{2}=C_{1} \backslash\left(S_{i_{2}, j_{2}}^{C_{1}} \backslash\left\{\left(s_{2}, t_{2}, l_{s_{2} t_{2}}\right)\right\}\right) \subseteq C_{1}$, being $\Theta_{2} \in \mathfrak{F}$ such that $L_{s_{2}, t_{2}}^{\Theta_{2}}=L_{i_{2}, j_{2}}$. Let $P_{2}=C_{2}^{\Theta_{2}} \subseteq C_{1}^{\Theta_{2}}$. By construction, $C_{1} \subseteq \mathfrak{F}\left(P_{2}\right)$ and so, $P_{1} \subseteq \mathfrak{F}\left(C_{1}\right) \subseteq \mathfrak{F}\left(\mathfrak{F}\left(P_{2}\right)\right)=\mathfrak{F}\left(P_{2}\right)$. Finally, $C \subseteq \mathfrak{F}\left(P_{1}\right) \subseteq \mathfrak{F}\left(\mathfrak{F}\left(P_{2}\right)\right)=\mathfrak{F}\left(P_{2}\right)$ Therefore, $\operatorname{scs}_{\mathfrak{F}}(L) \leq\left|P_{2}\right|=\left|C_{2}\right|<\left|C_{1}\right|$. We repeat all this procedure until we find that the maximum cardinality of all the corresponding sets $S_{i, j}$ is one.

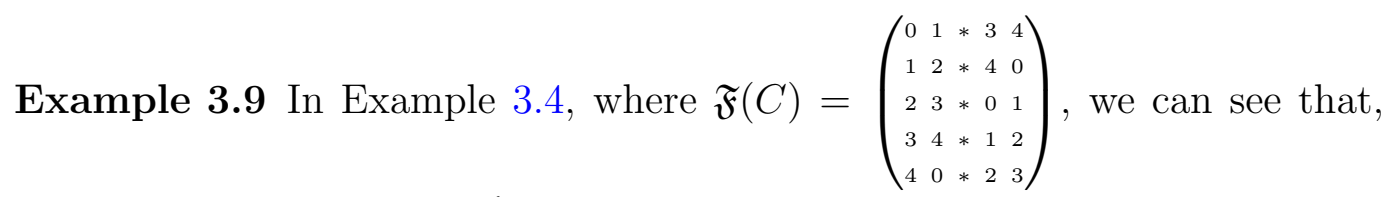
for all $i \in N,\left|S_{i, j}^{C}\right|=\left\{\begin{array}{l}1, \text { if } j=1 \text { or } 3, \\ 2, \text { if } j=0 \text { or } 4 .\end{array}\right.$. Let us then take for example $S_{0,0}^{C}=\{(0,0,0),(1,0,0)\}$ and let us consider $(0,0,0) \in S_{0,0}^{C}$. So, $C_{1}=$ 


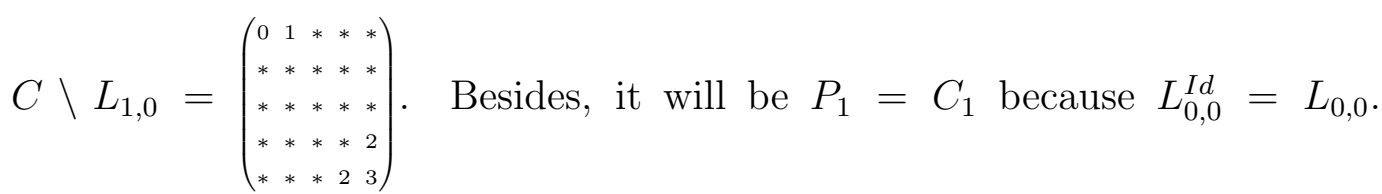
So, $C \subseteq \mathfrak{F}\left(P_{1}\right)=\mathfrak{F}(C)$ and $s c s_{\mathfrak{F}}(L) \leq\left|C_{1}\right|=5$. Now, for all $i \in N$, $\left|S_{i, j}^{C_{1}}\right|=\left\{\begin{array}{l}1, \text { if } j=0,1 \text { or } 3, \\ 2, \text { if } j=4 .\end{array}\right.$. Let us take $S_{3,4}^{C_{1}}=\{(3,4,2),(4,4,3)\}$ and

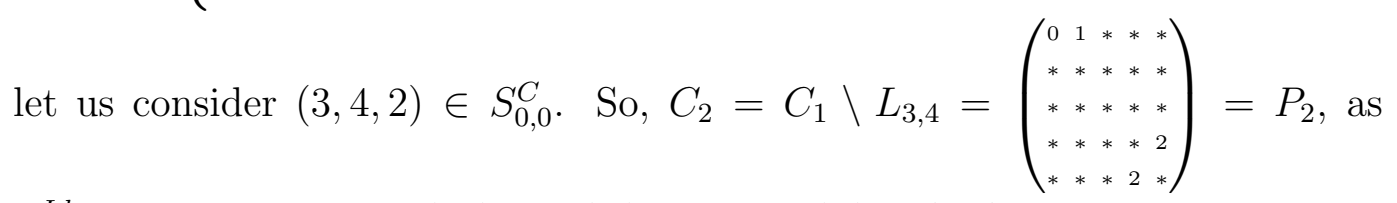
$L_{3,4}^{I d}=L_{3,4}$. So, $C \subseteq \mathfrak{F}\left(P_{2}\right)=\mathfrak{F}(C)$ and $\operatorname{scs}_{\mathfrak{F}}(L) \leq\left|C_{1}\right|=4$, as we have seen in Example 3.4. Thus, $S_{i, j}^{C_{2}}=1$ for all $i, j$ and so, the algorithm finishes. $\triangleleft$

\section{References}

[1] B. D. McKay, A. Meynert and W. Myrvold, Small Latin Squares, Quasigroups and Loops, Journal of Combinatorial Designs 15 (2007) 98-119. 